\title{
FACTORIZATIONS OF OUTER FUNCTIONS AND EXTREMAL PROBLEMS
}

\author{
by TAKAHIKO NAKAZI*
}

(Received 22nd November 1994)

\begin{abstract}
The author has proved that an outer function in the Hardy space $H^{1}$ can be factored into a product in which one factor is strongly outer and the other is the sum of two inner functions. In an endeavor to understand better the latter factor, we introduce a class of functions containing sums of inner functions as a special case. Using it, we describe the solutions of extremal problems in the Hardy spaces $H^{p}$ for $1 \leqq p<\infty$.
\end{abstract}

1991 AMS Subject classification: Primary 30D55, 46J15, secondary 47B35

\section{Introduction}

$N, N_{+}$and $H^{p}$ for $1 \leqq p<\infty$ denote the Nevanlinna class, the Smirnov class and the Hardy space, respectively on the open unit disc $U$ in the complex plane. A function $h$ in $N_{+}$is called outer if it is not divisible in $N_{+}$by a non-constant inner function. A function $g$ in $H^{1}$ is called strongly outer if the only functions $f$ in $H^{1}$ such that $f / g$ is non-negative are scalar multiples of $g$. If $g$ is not outer and so $g=q h$ for some inner $q$, then $f=(1+q)^{2} h$ belongs to $H^{1}$ and $f / g=(1+q)^{2} / q$ is non-negative. A norm one function in $H^{1}$ is outer if and only if it is an extreme point of the unit ball of $H^{1}[2]$. On the other hand, a norm one function in $H^{1}$ is strongly outer if and only if it is an exposed point of the unit ball of $H^{1}$ (cf. $[2,12]$. Like outer functions, strongly outer functions appear in many important areas, for example, function theory, operator theory and prediction theory.

It is not difficult to give a characterization of a strongly outer function similar to the above definition of an outer function. If $g$ is divisible in $H^{1}$ by a sum of two inner functions $q_{1}, q_{2}$ where $q_{1}+q_{2}$ is not constant and $\operatorname{Im} \bar{q}_{1} q_{2} \leqq 0$ almost everywhere, then $f=-i\left(q_{1}-q_{2}\right) g /\left(q_{1}+q_{2}\right)$ is not a scalar multiple of $g$ and $f / g$ is non-negative because $-i\left(q_{1}-q_{2}\right) /\left(q_{1}+q_{2}\right) \geqq 0$ almost everywhere. Thus $g$ is not strongly outer. The converse is also true by the following factorization theorem [12].

Theorem. If an outer function $h$ in $H^{1}$ is not strongly outer, then $h=\left(q_{1}+q_{2}\right)$ g where both $q_{1}$ and $q_{2}$ are inner, Im $\bar{q}_{1} q_{2} \leqq 0$ almost everywhere, $\left(q_{1}-q_{2}\right)^{-1}$ is summable and $g$ is strongly outer. If $q_{1}$ is a finite Blaschke product of degree $n$ then so is $q_{2}$.

*This research was partially supported by Grant-in-Aid for Scientific Research, Ministry of Education. 
The aim of this paper is to gain a better understanding of this theorem and of the sum of two inner functions. The sum of two inner functions appeared in H. Helson's papers [7] and [8]. D. Sarason [15] examined cases in which the sum of two nonconstant inner functions is outer. In this paper, we introduce functions in $H^{2}$ which have the form; $k=s+q \bar{s}$ where $s$ is in $L^{2}$ and $q$ is inner. If $s=1$, then $k=1+q$. If $s=q_{1}$ and $q=q_{1} q_{2}$ where $q_{1}$ and $q_{2}$ are inner, then $k=q_{1}+q_{2}$. If $f$ is the square of $H^{2}$ function $s+q \bar{s}$, then put $q_{1}=$ the inner part of $f+i q$ and $q_{2}=$ the inner part of $f-i q$. Then $\operatorname{Im} \bar{q}_{1} q_{2} \leqq 0, q_{1}+q_{2}$ is non-constant and $f$ is divisible in $H^{1}$ by $q_{1}+q_{2}$. By the remark above the Theorem, $f$ is not strongly outer. The following factorization theorem can be proved easily by a theorem of E. Hayashi $([5,6])$.

Theorem. If an outer function $h$ in $H^{1}$ is not strongly outer, then $h=(s+q \bar{s})^{2} g$ where $q$ is a non-constant inner function, $s+q \bar{s}$ is in $H^{2}$ and $g$ is strongly outer.

Proof. Suppose $h=k^{2}$ and $k$ is outer in $H^{2}$. By a theorem of E. Hayashi $([4,5])$,

$$
H^{2} \cap(k / \bar{k}) \bar{H}^{2}=g_{0}\left(H^{2} \ominus z q H^{2}\right)
$$

and $k / \bar{k}=\bar{q} \bar{g}_{0} / g_{0}$ where $q$ is inner and $g_{0}^{2}$ is strongly outer. Hence $k=l_{0}$ where $l \in H^{2} \ominus q z H^{2}$ and $\bar{q} l^{2} \geqq 0$. Put $s=l / 2$, then $l=s+q \bar{s}$ and $h=l^{2} g_{0}^{2}$.

In this theorem, we should like to be able to choose $s+q \bar{s}=q_{1}+q_{2}$ for some inner functions $q_{1}$ and $q_{2}$. Unfortunately we could not do except in some special cases [12]. Note that by an example of J. Inoue [9], we cannot choose $s+q \bar{s}=1+q$.

\section{Bad parts of outer functions}

In this section we study a function in $H^{2}$ which has the form $s+q \bar{s}$ where $s$ is in $L^{2}$ and $q$ is an inner function. If $\prod_{j=1}^{n}\left(q_{j}+q_{j}^{\prime}\right)$ where $q_{j}$ and $q_{j}^{\prime}$ are inner functions for $1 \leqq j \leqq n$, then $\prod_{j=1}^{n}\left(q_{j}+q_{j}^{\prime}\right)=s+q \bar{s}$ for $q=\prod_{j=1}^{n} q_{j} q_{j}^{\prime}$. Two natural questions are the following: (1) When is $s+q \bar{s}$ an outer function? (2) When can $s+q \bar{s}$ be divisible in $H^{2}$ by $1+q^{\prime}$ where $q^{\prime}$ denotes some nonconstant inner function? The question (1) is related with a paper of D. Sarason [15]. He studied it when $s+q \bar{s}$ is a sum of two inner functions. The question (2) is related with a paper of J. Inoue [9]. By the second theorem in the Introduction, Inoue's result is the following: There exists an outer function $f$ in $H^{2}$ which is not divisible in $H^{2}$ by any nonconstant $1+q^{\prime}$ but is divisible in $H^{2}$ by some nonconstant $s+q \bar{s}$, where $q$ and $q^{\prime}$ are inner functions. Because of the first theorem in the Introduction, we are also interested in nonconstant outer function $q_{1}+q_{2}$ such that both $q_{1}$ and $q_{2}$ are inner functions, $\operatorname{Im} \bar{q}_{1} q_{2} \leqq 0$ almost everywhere and $\left(q_{1}-q_{2}\right)^{-1}$ is summable.

Proposition 1. Suppose $s$ is a nonnegative function in $N_{+}$and $s^{-1}$ is summable. If $i-s=q_{1} l$ where $q_{1}$ is an inner function and $l$ is an outer function, then $q_{2}=(i+s / i-s) q_{1}$ is an inner function, $q_{1}+q_{2}$ is an outer function, $\operatorname{Im} \bar{q}_{1} q_{2} \leqq 0$ almost everywhere and 
$\left(q_{1}-q_{2}\right)^{-1}$ is summable. If $s$ is a rational function, then both $q_{1}$ and $q_{2}$ are finite Blaschke products of the same degree.

Proof. Since $\left|q_{2}\right|=1$ a.e. on $\partial U$ and $q_{2}=(i+s) / l, q_{2}$ is inner. Since $q_{1}+q_{2}=2 i l$, $q_{1}+q_{2}$ is outer. By a simple calculation,

$$
\frac{-\operatorname{Im} \bar{q}_{1} q_{2}}{\left|q_{1}+q_{2}\right|^{2}}=\frac{-i\left(q_{1}-q_{2}\right)}{q_{1}+q_{2}}=s \geqq 0 \quad \text { a.e. }
$$

and so $\operatorname{Im} \bar{q}_{1} q_{2} \leqq 0$ a.e. on $\partial U$. Since $\left(q_{1}-q_{2}\right)^{-1}=(i-s) /(-2 s)$ and $s^{-1}$ is summable, $\left(q_{1}-q_{2}\right)^{-1}$ is summable. If $s$ is a rational function, by [7] the number of zeros of $s-i$ and that of $s+i$ are equal. Hence $q_{1}$ and $q_{2}$ are finite Blaschke products of the same degree.

In Proposition 1 , if $s=-z /(1-z)^{2}$, then $q_{1}$ and $q_{2}$ have degree one. However even if $q_{1}$ and $q_{2}$ have degree one and $q_{1}+q_{2}$ is outer, $\operatorname{Im} \bar{q}_{1} q_{2}$ is not necessarily non-negative. In fact, suppose $|a|<1$ and $|\rho|=1$. Then, $\rho z+\bar{\rho}(z-a / 1-\bar{a} z)$ is outer if and only if $|\operatorname{Re} \rho| \leqq|a|,[15]$. However $\operatorname{Im} \bar{z}(z-a / 1-\bar{a} z)$ is not non-negative on $\partial U$.

Proposition 2. Suppose $s+q \bar{s}$ is in $H^{2}$, where $q$ is an inner function and $s$ is in $L^{2}$. Then $s+q \bar{s}$ is an outer function if and only if there exists a function $t$ in $L^{2}$ such that $s+(t-q \bar{t})$ is an outer function.

Proof. If $l=s+(t-q \bar{t})$ is outer, then $s+q \bar{s}=l+q T \in H^{2}$ and $q \bar{l} \in H^{2}$. Hence $q \bar{l}=q_{0} l$ for some inner function $q_{0}$. Then $s+q \bar{s}=l\left(1+q(\bar{l})=l\left(1+q_{0}\right)\right.$ and hence $s+q \bar{s}$ is outer. Conversely if $s+q \bar{s}=2 l$ is outer, then $q T=l$ and hence $s+q \bar{s}=l+q I$. Let $k=l-s$, then $k+q \bar{k}=0$ and so $k=t-q \bar{t}$, where $t=k / 2$. Thus $l=s+(t-q \bar{t})$ is outer.

Corollary 1. Suppose $s+q \bar{s}$ is in $H^{2}$, where $q$ is an inner function and $s$ is in $L^{2}$. If $s$ and $q$ satisfy one of the following (1) $\sim(3)$, then $s+q \bar{s}$ is an outer function.

(1) $s$ is an outer function.

(2) $q=q_{1} q_{2}$ and $s=q_{1} h$ where $q_{1}$ and $q_{2}$ are inner functions, $h$ is an outer function and $q_{2} \bar{h}=\alpha$ for some complex number $\alpha$.

(3) $q=q_{1} q_{2}$ and $s=q_{1} h$ where $\left\{q_{j}\right\}_{j=1,2,3}$ are inner functions, $h$ is an outer function, $q_{2} \bar{h}=q_{3} h$, and $q_{1}+q_{3}$ is an outer function.

Proof. (1) is clear by Proposition 2 and (2) is a special case of (3). For (3), let $t=\left(q_{3}-q_{1}\right) h / 4$, then

$$
q \bar{t}=\frac{1}{4} q\left(\bar{q}_{2} h-\bar{q}_{1} \bar{h}\right)=\frac{1}{4}\left(q_{1} h-q_{2} \bar{h}\right)=\frac{1}{4}\left(q_{1}-q_{3}\right) h
$$

because $q_{2} \bar{h}=q_{3} h$. Hence $t-q \bar{t}=\left(q_{3}-q_{1}\right) h / 4$ and so $s+(t-q \bar{t})=\left(q_{3}+q_{1}\right) h / 2$. This implies (3) because $q_{1}+q_{3}$ is outer. 
Proposition 3. Suppose $q_{1}$ is an inner function and $s+q \bar{s}$ is a non-zero function in $H^{2}$, where $q$ is an inner function and $s$ is in $L^{2}$. Then $s+q \bar{s}$ is divisible in $H^{2}$ by $1+q_{1}$ if and only if there exists a function $t$ in $L^{2}$ such that $q\{\bar{s}+(\bar{t}-q \bar{t})\}=q_{1}\{s+(t-q \bar{t})\}$. In particular, if $q \bar{s}=q_{1} s$ then $s+q \bar{s}$ is divisible by $1+q_{1}$.

Proof. If there exists a function $t$ in $L^{2}$ such that $q\{\bar{s}+(\bar{t}-\bar{q} t)\}=q_{1}\{s+(t-q \bar{t})\}$, then $s+q \bar{s}=s+t-q \bar{t}+q(\bar{s}+\bar{t}-\bar{q} t)=s+t-q \bar{t}+q_{1}(s+t-q \bar{t})=(s+t-q \bar{t})\left(1+q_{1}\right)$ and hence $s+$ $q \bar{s}$ is divisible in $H^{2}$ by $1+q_{1}$. Conversely if $l=(s+q \bar{s}) /\left(1+q_{1}\right)$ is in $H^{2}$, then

$$
\bar{q}=\frac{\bar{s}+\bar{q} s}{s+q \bar{s}}=\frac{T}{l} \frac{1+\bar{q}_{1}}{1+q_{1}}=\frac{T}{l} \bar{q}_{1}
$$

and hence $q T=q_{1} l$. If $k=l-s$, then $k=t-q \bar{t}$ for some $t \in L^{2}$ and hence $l=s+(t-q \bar{t})$. This implies that $q\{\bar{s}+(\bar{t}-\bar{q} t)\}=q_{1}\{s+(t-q \bar{t})\}$.

Corollary 2. Suppose $s+q \bar{s}$ is in $H^{2}$, where $q$ is an inner function and $s$ is in $L^{2}$.

(1) If $s$ is an outer function and $q \bar{s} \neq \alpha s$ for any $\alpha$ in $C$ with $|\alpha|=1$, then there exists $a$ non-constant inner function $q_{1}$ such that $s+q \bar{s}$ is divisible in $H^{2}$ by $1+q_{1}$.

(2) If $h$ is an outer function, $q \bar{h}=q_{1} q_{2}^{2} h$ and $s=q_{2} h$ where $q_{1}$ and $q_{2}$ are inner functions, then $s+q \bar{s}$ is divisible in $H^{2}$ by $1+q_{1}$.

(3) If $q$ is a finite Blaschke product, then there exists a non-constant finite Blaschke product $q_{1}$ such that $s+q \bar{s}$ is divisible in $H^{2}$ by $1+q_{1}$, or $s+q \bar{s}$ is not an outer function.

Proof. (1) Since $s$ is outer, $q \bar{s}=q_{1} s$ for some inner function $q_{1}$. By the hypothesis, $q_{1}$ is non-constant and hence Proposition 3 implies (1).

(2) $q \bar{s}=q_{1} q_{2} h=q_{1} s$ implies (2) by Proposition 3 .

(3) Since $\bar{q}(s+q \bar{s})^{2} \geqq 0$ a.e. on $\partial U$ and $q$ is a finite Blaschke product, $(s+q \bar{s})^{2}=$ $\prod_{j=1}^{n}\left(z-a_{j}\right)\left(1-\bar{a}_{j} z\right) l^{2}$, where $\left|a_{j}\right| \leqq 1(1 \leqq j \leqq n)$ and $l$ is outer in $H^{2}([2,11])$. Therefore if $s+q \bar{s}$ is outer, then $s+q \bar{s}=\prod_{j=1}^{n}\left(-\bar{a}_{j}\right)^{1 / 2}\left(z-a_{j}\right) l$ and $\left|a_{j}\right|=1$. Thus $s+q \bar{s}$ is divisible in $H^{2}$ by $z-a_{j}$.

When $q_{1}$ and $q_{2}$ are inner functions, we write $q_{1}<q_{2}$ if there exists a nonzero function $f$ in $H^{1}$ such that $\bar{q}_{1} q_{2}=f /|f|$. If both $q_{1}$ and $q_{2}$ are finite Blaschke product, then $q_{1} \prec q_{2}$ is equivalent to (degree of $q_{1}$ ) $\leqq\left(\right.$ degree of $q_{2}$ ). For each $g$ in $H^{1}$, sing $g$ denotes the set of the unit circle on which $g$ cannot be analytically extended.

Proposition 4. If $q_{1}$ and $q_{2}$ are inner functions and the inner part of $q_{1}+q_{2}$ is $q$, then $q<q_{1}$ and $q<q_{2}$.

Proof. Let $q_{1}+q_{2}=q h$, then $\left|\bar{q} q_{1}-h\right|=\left|\bar{q} q_{2}-h\right|=1$. By a theorem of P. Koosis (cf. [4, Chapter 4, Lemma 5.4]), $q \prec q_{1}$ and $q \prec q_{2}$. 
Corollary 3. Suppose $q_{1}$ and $q_{2}$ are inner functions and $q_{1}+q_{2}=q h$ where $q$ is an inner function and $h$ is an outer function.

(1) If $q_{1}$ is a finite Blaschke product, then $q$ is also a finite Blaschke product and (degree of q) $\leqq\left(\right.$ degree of $\left.q_{1}\right)$.

(2) If (sing $\left.q_{1}\right) \cap\left(\operatorname{sing} q_{2}\right)$ is empty, then $q$ is a finite Blaschke product.

(3) Suppose $q_{1}=\exp (-(a+z) /(a-z))$ and $q_{2}=-\alpha \exp (-(b+z) /(b-z))$, where $|a|=|b|=$ $1, b=-\bar{a}$ and $|\alpha|=1$. If $\alpha=1$, then $q=z$ or $q$ is constant. If $\alpha \neq 1$, then $q$ is always constant, that is, $q_{1}+q_{2}$ is an outer function.

Proof. (1) By Proposition 4, $\bar{q} q_{1}=f /|f|$ for some function $f \in H^{1}$ and hence $\bar{q}_{1}(q f) \geqq 0$ a.e. on $\partial U$. If $q_{1}$ is a finite Blaschke product of degree $m, q f=$ $\prod_{j=1}^{n}\left(z-a_{j}\right)\left(1-\bar{a}_{j} z\right) l$ and $n \leqq m$ where $\left|a_{j}\right| \leqq 1(1 \leqq j \leqq n)$ and $l$ is strongly outer. Hence $q$ is a finite Blaschke product of degree $k$ and $k \leqq n$.

(2) By Proposition $4, \bar{q} q_{1}=f /|f|$ for some function $f \in H^{1}$ and hence $\bar{q} q_{1}=g / \bar{g}$ for some outer function $g \in H^{2}$. Therefore $\bar{q}_{1} q g=\bar{g}$ and so $q g \in H^{2} \ominus q_{1} z H^{2}$. Hence sing $q_{1} \supseteq$ sing $q g$ and by [10, Lemma 4], sing $q_{1} \supseteq \operatorname{sing} q$. Similarly sing $q_{2} \supseteq \operatorname{sing} q$ and by the hypothesis $q$ is a finite Blaschke product.

(3) By (2), $q$ is a finite Blaschke product. If $q(x)=0$ for some point $x \in U$, then $\exp (-(a+x) /(a-x))=\alpha \exp (-(b+x) /(b-x))$ and hence

$$
-\frac{a+x}{a-x}=-\frac{b+x}{b-x}+i \rho \text { and } \rho=t+2 n \pi
$$

where $n$ is some integer and $\alpha=e^{i t}$. If $\rho=0$ then $q=z$ because $a \neq b$. Suppose $\rho \neq 0$. Then

$$
x^{2}-\left\{\left(1-\frac{2 i}{\rho}\right) b+\left(1+\frac{2 i}{\rho}\right) a\right\} x+a b=0
$$

If $A$ and $B$ are the solutions of the above quadratic equation, then $A B=a b=-1$ and

$$
A+B=\left(1+\frac{2 i}{\rho}\right) a-\left(1+\frac{2 i}{\rho}\right) a
$$

This implies $|A|=|B|=1$ and contradicts $|x|<1$.

(1) of Corollary 3 was proved by D. Sarason [15, Proposition 3]. Our proof is different from his.

\section{Projection}

For each inner function $q$, we define two operators on $L^{2}$ 


$$
L_{q}(s)=\frac{s+q \bar{s}}{2} \text { and } \quad L_{q}^{\prime}(s)=\frac{s-q \bar{s}}{2}
$$

If $q=1$, then $L_{q}(s)$ is the real part of $s$ and $L_{d}^{\prime}(s)$ is the imaginary part of $s$. In general, $\left|L_{q}(s)\right| \leqq|s|$ and $\left|L_{q}^{\prime}(s)\right| \leqq|s|$. Hence $L_{q}$ and $L_{q}^{\prime}$ are contractive. $L_{q}$ and $L_{q}^{\prime}$ commute with multiplication operators by real valued functions in $L^{\infty}$. Moreover on $L^{2}$, we have $L_{q} L_{q}=L_{q}$ and $L_{q} L_{q}^{\prime}=0$ and $L_{q}+L_{q}^{\prime}$ is the identity operator. By results of the last section, we are interested in a function $s$ such that $L_{q}(s)$ belongs to $H^{2}$. Since $q=(1+q)^{2} /|1+q|^{2}$, we define $q^{1 / 2}=(1+q) /|1+q|$. Put

$$
6 \mathscr{A}_{q}=\left\{g \in H^{2}: \frac{g}{1+q} \text { is a real valued function }\right\}
$$

Theorem 5. Let $q$ be a non-constant inner function. Then

$$
\left\{s \in L^{2}: L_{q}(s) \in H^{2}\right\}=\mathscr{A}_{q}+i q^{1 / 2} L_{R}^{2}
$$

where $L_{R}^{2}=\left\{s \in L^{2}\right.$ : $s$ is a real valued function $\}$. In particular, if $s+q \bar{s}$ belongs to $H^{2}$ for some $s$ in $L^{2}$, then $s+q \bar{s}=t+q \bar{t}$ for some $t$ in $H^{2}$.

Proof. If $g \in \mathscr{A}_{q}$ then $u=g /(1+q)$ is real and $g=u(1+q)$. Hence $q \bar{g}=g$ and so $L_{q}(g)=g \in H^{2}$. If $s=i q^{1 / 2} u$ and $u \in L_{R}^{2}$ then $L_{q}(s)=0$. This implies that $\left\{s \in L^{2}: L_{q}(s) \in H^{2}\right\}$ $\supseteq \mathscr{A}_{q}+i q^{1 / 2} L_{R}^{2}$. Conversely, suppose $g=L_{q}(s) \in H^{2}$. If $g=0$, then $s=-q \bar{s}$ and $s^{2}=$ $-q|s|^{2}$. Hence $\left(i \bar{q}^{1 / 2} s\right)^{2}=-\bar{q} s^{2}=|s|^{2} \geqq 0$ and so $i \bar{q}^{1 / 2} s=-u$ is real. Thus $s=i q^{1 / 2} u$ and $u \in L_{R}^{2}$. If $g \neq 0, s+q \bar{s}=2 g$ and

$$
\frac{s}{g}+\left(\frac{s}{g}\right)=2
$$

Put $t=s / g-1$, then $t+\bar{t}=0$ and so $t=i v$ for some $v \in L_{R}^{2}$. Hence $s=g+i v g$ and $v g=q^{1 / 2} u$, where $u=v \bar{q}^{1 / 2} g$ is in $L_{R}^{2}$. Thus $s=g+i q^{1 / 2} u$. This completes the proof of the theorem.

Corollary 4. Let $q$ be a non-constant inner function. Then

$$
\left\{s \in H^{2}: L_{q}(s) \in H^{2}\right\}=\mathscr{A}_{q}+i \mathscr{A}_{q}
$$

and hence $H^{2} \ominus q z H^{2}=\mathscr{A}_{q}+i \mathscr{A}_{q} . L_{q}$ is the projection from $H^{2} \ominus q z H^{2}$ onto $\mathscr{A}_{q}$ and has kernel $i \mathscr{A}_{\mathbf{q}}$.

Proof. If $g \in \mathscr{A}_{q}$, then $g=v(1+q)$ for some real valued function $v$ and so $g=q^{1 / 2} u$ 
where $u=v|1+q|$. Hence $\mathscr{A}_{q} \subset q^{1 / 2} L_{R}^{2}$ and $\left(q^{1 / 2} L_{R}^{2}\right) \cap H^{2}=\mathscr{A}_{q}$. Now Theorem 5 implies the corollary.

The proof of Theorem 5 is related to that of [14, Theorem 3]. The equality in Corollary 4, that is, $H^{2} \ominus q z H^{2}=\mathscr{A}_{q}+i \mathscr{A}_{q}$ is known by [12,(1) of Theorem 3].

Corollary 5. Let $q$ be an inner function.

(1) If $q=z^{n}$, then $\mathscr{A}_{q}=\left\{\sum_{j=0}^{n} b_{j} z^{j}: b_{j}=\bar{b}_{n-j}\right\}$.

(2) If $q=\prod_{l=1}^{\infty}\left(-\bar{a}_{l} /\left|a_{l}\right|\right)\left(z-a_{l} / 1-\bar{a}_{l} z\right)$ and $\sum_{l=1}^{\infty}\left(1-\left|a_{l}\right|\right)<\infty$, then

$$
\mathscr{A}_{q}=\left\{\sum_{j=0}^{\infty} \frac{c_{j} B_{j}+\bar{c}_{j} z B_{j}^{\prime}}{1-\bar{a}_{j} z}: \sum_{j=0}^{\infty} \frac{\left|c_{j}\right|^{2}}{\left(1-\left|a_{j}\right|\right)^{2}\left(1+\left|a_{j}\right|\right)}<\infty\right\}
$$

where $\quad B_{j}=\prod_{l=1}^{j-1}\left(-\bar{a}_{j} /\left|a_{j}\right|\right)\left(z-a_{j} / 1-\bar{a}_{j} z\right), \quad B_{j}^{\prime}=\prod_{l=j}^{\infty}\left(-\bar{a}_{j} /\left|a_{j}\right|\right)\left(z-a_{j} / 1-\bar{a}_{j} z\right), \quad a_{0}=0$, $B_{0}=1$ and $B_{0}^{\prime}=q$.

Proof. (1) If $s \in H^{2} \Theta q z H^{2}$, then $s=\sum_{j=0}^{n} a_{j} z^{j}$ and hence $s+q \bar{s}=\sum_{j=0}^{n}\left(a_{j}+\bar{a}_{n-j}\right) z^{j}$. Now corollary 4 implies (1).

(2) If $s \in H^{2} \ominus q z H^{2}$, then by [1]

$$
s=\sum_{j=0}^{\infty} c_{j}\left(1+\left|a_{j}\right|\right)^{1 / 2} B_{j}\left(1-\bar{a}_{j} z\right)^{-1}\left(1-\left|a_{j}\right|\right)
$$

and $\sum_{j=0}^{\infty}\left|c_{j}\right|^{2}<\infty$. Hence

$$
\begin{aligned}
s+q \bar{s} & =\sum_{j=0}^{\infty}\left(c_{j} \frac{B_{j}}{1-\bar{a}_{j} z}+\bar{c}_{j} \frac{q \bar{B}_{j}}{1-\bar{a}_{j} z}\right)\left(1+\left|a_{j}\right|\right)^{1 / 2}\left(1-\left|a_{j}\right|\right) \\
& =\sum_{j=0}^{\infty}\left(\frac{c_{j} B_{j}+\bar{c}_{j} z B_{j+1}^{\prime}}{1-\bar{a}_{j} z}\right)\left(1+\left|a_{j}\right|\right)^{1 / 2}\left(1-\left|a_{j}\right|\right) .
\end{aligned}
$$

Now Corollary 4 implies (2).

A theorem of P. R. Ahern and D. N. Clark [1, Theorem 3.1], lets one describe $\mathscr{A}_{q}$ for arbitrary inner function $q$.

\section{Extremal problems}

Let $1 \leqq q \leqq \infty$ and $1 / p+1 / l=1$. If $\phi \in L^{l}$, we denote by $T_{\phi}^{p}$ the continuous functional defined on the Hardy space $H^{p}$ by 


$$
T_{\phi}^{p}(f)=\int_{-\pi}^{\pi} f\left(e^{i \theta}\right) \phi\left(e^{i \theta}\right) d \theta / 2 \pi
$$

A function $f$ in $H^{p}$, which satisfies $T_{\phi}^{p}(f)=\left\|T_{\phi}^{p}\right\|$ and $\|f\|_{p} \leqq 1$, is called an extremal function. A function $\phi$ in $L^{\prime}$ is called an extremal kernel when $\|\phi\|_{l}=\left\|T_{\phi}^{p}\right\|$. The existence and uniqueness of extremal functions and extremal kernels is known for $1<p \leqq \infty$ (cf. [3, Theorem 8.1]). For $p=1$, the situation is very different. An extremal function may not exist, the dual extremal kernel always exists and is unique if an extremal function exists (cf. [3, Theorem 8.1]). For $p=1$, the set $S_{\phi}$ of all extremal functions is defined by

$$
S_{\phi}=\left\{f \in H^{1}: T_{\phi}^{1}(f)=\left\|T_{\phi}^{1}\right\| \text { and }\|f\|_{1}=1\right\}
$$

$S_{\phi}$ has been described in general by E. Hayashi $[5,6]$. In this section, we describe $S_{\phi}$ completely in ways different from that of E. Hayashi. Moreover using the result we describe extremal kernels and extremal functions for $1<p<\infty$.

Theorem 6. Suppose $p=1$ and $S_{\phi}$ is nonempty. Then there exist an inner function $q$ and a strong outer function $g$ which satisfy the following (1) (4).

(1) The unique extremal kernel of $T_{\phi}^{1}$ is $\bar{q}|g| / g$.

(2) $f$ is a member of $S_{\phi}$ if and only if

$$
f=\gamma q_{0}\left(\frac{s+q \bar{s}}{1+q_{0}}\right)^{2} g
$$

where $\gamma$ is a positive constant, $\|f\|_{1}=1, q_{0}$ is an inner function, $s$ is in $H^{2} \ominus q z H^{2}$ and $(s+q \bar{s}) /\left(1+q_{0}\right)$ is an outer function in $H^{2}$.

(3) $f$ is a member of $S_{\phi}$ if and only if

$$
f=\gamma q_{0}\left(t+q \overline{q_{0} t}\right)^{2} g
$$

where $\gamma$ is a positive constant, $\|f\|_{1}=1, q_{0}$ is an inner function, $t$ is in $H^{2} \ominus q z H^{2}$ and $t+q \overline{q_{0} t}$ is an outer function in $H^{2} \Theta q z H^{2}$.

(4) $f$ is a member of $S_{\phi}$ if and only if

$$
f=\gamma\left\{(s+q \bar{s})^{2}+(t+q \bar{t})^{2}\right\} g
$$

where $\gamma$ is a positive constant, $\|f\|_{1}=1$, and $s$ and $t$ are in $H^{2} \Theta q z H^{2}$.

Proof. (1) is known from [5].

(2) If $f=\gamma q_{0}\left(s+q \bar{s} / 1+q_{0}\right)^{2} g$, then 


$$
\frac{|f|}{f}=q_{0} \frac{\left|1+q_{0}\right|^{2}}{\left(1+q_{0}\right)^{2}} \frac{|s+q \bar{s}|^{2}}{(s+q \bar{s})^{2}} \frac{|g|}{g}=\bar{q} \frac{|g|}{g}
$$

and hence $f \in S_{\phi}$. Conversely, if $f \in S_{\phi}$ and $f=q_{0} h^{2}$, where $q_{0}$ is inner and $h$ is outer, then $\gamma_{1}\left(1+q_{0}\right)^{2} h^{2} \in S_{\phi}$ for some positive constant $\gamma_{1}$. Since $\left(1+q_{0}\right) h$ is outer in $H^{2}$, by a theorem of $\mathrm{E}$. Hayashi $([5,6])$,

$$
H^{2} \cap q_{0}(h / \bar{h}) \bar{H}^{2}=q_{0}\left(H^{2} \ominus q z H^{2}\right)
$$

and $q_{0}(h / \bar{h})=\bar{q} \bar{q}_{0} / q_{0}$, where $q$ is inner and $g=g_{0}^{2}$, is strongly outer. Hence $\left(1+q_{0}\right) h=k g_{0}$ where $k \in H^{2} \Theta q z H^{2}$ and $\bar{q} k^{2} \geqq 0$. Since $k \in \mathscr{A}_{q}$, by Corollary $4, k=s+q \bar{s}$ for some function $s \in H^{2} \Theta q z H^{2}$. Now $q_{0} h$ belongs to $g_{0}\left(H^{2} \Theta q z H^{2}\right)$ because $q_{0} h=q_{0}(k / \bar{h}) h$. Therefore $q_{0} h / g_{0}$ belongs to $H^{2} \ominus q z H^{2}$ and hence $h / g_{0}=(s+q \bar{s}) /\left(1+q_{0}\right)$ belongs to $N_{+} \cap L^{2}=H^{2}$. This implies (2).

(3) Put $(s+q \bar{s}) /\left(1+q_{0}\right)=l$ in (2); then

$$
\bar{l}=\frac{\bar{s}+\bar{q} s}{1+\bar{q}_{0}} \frac{1+q_{0}}{s+q \bar{s}}=q_{0} \bar{q} .
$$

Hence $l=q \overline{q_{0}} l$ and so $l=t+q \overline{q_{0}} t$, where $t=l / 2 \in H^{2}$. This implies (3).

(4) By (2), the if part is clear. Conversely if $f \in S_{\phi}$, then by (2) $f=q_{0} k^{2} g$, where $k=\gamma^{1 / 2}(s+q \bar{s}) /\left(1+q_{0}\right)$. Since $\bar{q} q_{0} k^{2}=|k|^{2}, q \bar{k}=q_{0} k$ and hence $k \in H^{2} \ominus q z H^{2}$. By Corollary $4, k=l+i m$ for some functions $l, m \in \mathscr{A}_{q}$ and hence $q_{1} k=l-i m$ for some inner function $q_{1}$. Thus $q_{1} k^{2}=l^{2}+m^{2}$ and hence $\bar{q} q_{1} k^{2}=|k|^{2}$. Therefore $q_{1}=q_{0}$. Corollary 4 implies (4) because $f=\gamma\left\{l^{2}+m^{2}\right\} g$.

If $(s+q \bar{s}) /\left(1+q_{0}\right)$ belongs to $H^{2}$, then $q_{0}<q$ and $(s+q \bar{s}) /\left(1+q_{0}\right)$ belongs to $H^{2} \ominus$ $q z H^{2}$. In fact, if $l=(s+q \bar{s}) /\left(1+q_{0}\right)$, then by the proof of (3) of Theorem $6, q I=q_{0} l$. Hence $l$ belongs to $H^{2} \Theta q z H^{2}$ and $q_{0}<q$ because $\bar{q}_{0} q=l^{2} /|l|^{2}$. Theorem 7 and Theorem 1 in [13] describe extremal kernels and extremal functions in case $1<p<\infty$.

Theorem 7. Suppose $1<p<\infty$ and $1 / p+1 / l=1$. Then $\phi$ is the unique extremal kernel and $f$ is the unique extremal function of $T_{\phi}^{p}$ if and only if there exist an inner function $q$ and a strong outer function $g$ which satisfy the following:

and

$$
\phi=\left\|T_{\phi}^{p}\right\| \bar{q} \frac{|g|}{g}\left(\frac{s+q \bar{s}}{1+q_{0}}\right)^{2 / t} g^{1 / l}
$$

$$
f=q_{0}\left(\frac{s+q \bar{s}}{1+q_{0}}\right)^{2 / p} g^{1 / p},
$$

where $q_{0}$ is an inner function, $\|f\|_{p}=1,\|\phi\|_{l}=\left\|T_{\phi}^{p}\right\|, s \in H^{2} \Theta q z H^{2}$ and $(s+q \tilde{s}) /\left(1+q_{0}\right)$ is an outer function in $H^{2}$. 
Proof. If $\phi$ is the unique extremal kernel and $f$ is the unique extremal function of $T_{\phi}^{p}$, then by $[13$, Theorem 1$]$

and

$$
\phi=\phi_{0} h, f=\left\|T_{\phi}^{l}\right\|^{-l / p} Q h^{l / p}
$$

$$
\left\|T_{\phi}^{l}\right\|^{-l} Q h^{l} \in S_{\phi_{0}}, \phi_{0}=\bar{Q}|h|^{\prime} h^{-l} \text {, }
$$

where $h$ is outer with $|\phi|=|h|$ and $Q$ is the inner part of $f$. By Theorem 6,

$$
\left\|T_{\phi}^{l}\right\|^{-l} Q h^{l}=q_{0}\left(\frac{s+q \bar{s}}{1+q_{0}}\right)^{2} g,
$$

where $q$ and $q_{0}$ are inner, $g$ is strongly outer, $\left\|q_{o}\left(s+q \bar{s} / 1+q_{0}\right)^{2} g\right\|_{1}=1, s \in H^{2} \Theta q z H^{2}$ and $(s+q \bar{s}) /\left(1+q_{0}\right)$ is outer in $H^{2}$. Hence $Q=q_{0}, h=\left\|T_{\phi}^{l}\right\|\left(s+q \bar{s} / 1+q_{0}\right)^{2 / l} g^{1 / l}$ and $\phi_{0}=\bar{q}_{0}\left(|h|^{l} / h^{l}\right)=\bar{q}(|g| / g)$.

Thus

and

$$
\phi=\bar{q} \frac{|g|}{g}\left\|T_{\phi}^{l}\right\|\left(\frac{s+q \bar{s}}{1+q_{0}}\right)^{2 / l} g^{1 / l}
$$

$$
f=\left\|T_{\phi}^{l}\right\|^{-l / p} q_{0} h^{l / p}=q_{0}\left(\frac{s+q \bar{s}}{1+q_{0}}\right)^{2 / p} g^{1 / p} .
$$

Theorem 6 is a generalization of [11, Theorem 2]. Theorem 7 is a generalization of [13, Theorem 2]. But the descriptions are different from the previous ones. In those descriptions, the bad part $q_{\mathrm{o}}\left(s+q \bar{s} / 1+q_{0}\right)^{2 / 1}$ is important. If $f$ is an inner function, then it is clear that $\left\|f+\bar{z} \bar{H}^{l}\right\|=\|f\|_{l}$ for $1 \leqq l \leqq \infty$. If $f=q_{0}\left(s+q \bar{s} / 1+q_{0}\right)^{2 / l}$, then, by Theorem $8,\left\|f+\bar{z} \bar{H}^{l}\right\|=\|f\|_{l}$ for $1 \leqq l \leqq \infty$. Theorem 8 also shows [13, Corollary 3]. To prove Theorem 8 we need the following lemma.

Lemma. Suppose $1 \leqq l \leqq \infty$ and $f=q h$ is in $H^{\prime}$, where $q$ is an inner function and $h$ is an outer function. Then $\left\|f+\bar{z} \bar{H}^{l}\right\|=\|f\|_{1}$ if and only if $q h^{2-l} /|h|^{2-l}$ is an inner function.

Proof. For $l \neq 1$ the lemma is known [13, Corollary 2]. Suppose $l=1$. By [3, p. 133], if $\left\|f+\bar{z} \bar{H}^{1}\right\|=\|f\|_{1}$, then there exists an extremal function $Q \in H^{\infty}$ and $|Q|=1$ a.e. on $\left\{\theta ; f\left(e^{i \theta}\right) \neq 0\right\}$ and $Q f \geqq 0$ a.e. on $\partial U$. Hence $Q$ is inner and so $f /|f|$ is inner. The converse is clear.

Theorem 8. Suppose $1 \leqq l \leqq \infty$ and $f$ is a nonzero function in $H^{l}$.

(1) $\left\|f+\bar{z} \bar{H}^{2}\right\|=\|f\|_{2}$ for an arbitrary function $f$ in $H^{2}$.

(2) For $2<l<\infty,\left\|f+\bar{z} \bar{H}^{l}\right\|=\|f\|_{\text {, }}$ if and only if 


$$
f=q\left(\frac{s+q \bar{s}}{1+Q}\right)^{2 / l-2}
$$

where $q$ and $Q$ are inner functions with $Q<q$.

(3) For $1 \leqq l<2,\left\|f+\bar{z} \bar{H}^{l}\right\|=\|f\|_{l}$ if and only if

$$
f=q\left(\frac{s+Q \bar{s}}{1+q}\right)^{2 / 2-l}
$$

where $q$ and $Q$ are inner functions with $q<Q$.

(4) Suppose $l=\infty$ and $S_{\bar{f}}$ is nonempty. Then $\left\|f+\bar{z} \bar{H}^{\infty}\right\|=\|f\|_{\infty}$ if and only if $f$ is an inner function.

Proof. (1) is clear because $f$ is orthogonal to $\bar{z} \bar{H}^{2}$. Suppose $f=q h$ where $q$ is inner and $h$ is outer.

(2) If $\left\|f+\bar{z} \bar{H}^{l}\right\|=\|f\|_{l}$, then by Lemma $q h^{2-l} /|h|^{2-l}=Q$ is inner. Hence $\bar{q} Q h^{l-2}=$ $\left|h^{l-2}\right|$. If $1<t<l / l-2$, then $h^{l-2} \in H^{t}$ and so $h^{l-2} \in H^{1}$. Now Theorem 6 implies that

$$
Q h^{l-2}=Q\left(\frac{s+q \bar{s}}{1+Q}\right)^{2} \text { and } Q \prec q .
$$

Hence $h=(s+q \bar{s} / 1+Q)^{2 / l-2}$ and so $f=q(s+q \bar{s} / 1+Q)^{2 / l-2}$. Conversely if $f=$ $q(s+q \bar{s} / 1+Q)^{2 / l-2}$, then $h=(s+q \bar{s} / 1+Q)^{2 / l-2}$ and hence

$$
\bar{q} \frac{h^{l-2}}{|h|^{l-2}}=\bar{q} \frac{(s+q \bar{s})^{2}}{(1+Q)^{2}} \frac{|1+Q|^{2}}{|s+q \bar{s}|^{2}}=Q
$$

The lemma implies $\left\|f+\bar{z} \bar{H}^{l}\right\|=\|f\|_{l}$.

(3) If $\left\|f+\bar{z} \bar{H}^{l}\right\|=\|f\|_{l}$, then by the lemma $q h^{2-l} /|h|^{2-l}=Q$ is inner. Hence $\bar{Q} q h^{2-l}=$ $|h|^{2-l}$ and $h^{2-l} \in H^{1}$ because $h^{l} \in H^{1}$ and $l>2-l>0$. Again by Theorem 6

$$
q h^{2-l}=q\left(\frac{s+Q \bar{s}}{1+q}\right)^{2} \text { and } q \prec Q
$$

Hence $h=(s+Q \bar{s} / 1+q)^{2 / 2-1}$ and so $f=q(s+Q \bar{s} / 1+q)^{2 / 2-l}$. Conversely if $f=$ $q(s+Q \bar{s} / 1+q)^{2 / 2-1}$, then

$$
\bar{q} \frac{h^{l-2}}{|h|^{l-2}}=\bar{q} \frac{(s+Q \bar{s})^{2}}{(1+q)^{2}} \frac{|1+q|^{2}}{|s+Q \bar{s}|^{2}}=Q
$$

The lemma implies $\left\|f+\bar{z} \bar{H}^{\prime}\right\|=\|f\|_{1}$. 
(4) If $S_{\bar{f}}$ is nonempty and $\left\|f+\bar{z} \bar{H}^{\infty}\right\|=\|f\|_{\infty}$, then $f$ is inner by Theorem 6 .

\section{REFERENCES}

1.P. R. Ahern and D. N. Clark, On functions orthogonal to invariant subspaces, Acta Math. 124 (1970), 191-204.

2. K. DELeEuw and W. Rudin, Extreme points and extremum problems in $H^{1}$, Pacific J. Math. 8 (1958), 467-485.

3. P. Duren, Theory of $H^{p}$ spaces (Academic Press, New York, 1970).

4. J. B. Garnett, Bounded analytic functions (Academic Press, 1981).

5. E. Hayashi, The solution sets of extremal problems in $H^{1}$, Proc. Amer. Math. Soc. 93 (1985), 690-696.

6. E. HAYASH, The kernel of a Toeplitz operator, Integral $E_{q}$. Oper. Theory 9 (1986), 589-591.

7. H. Helson, Large analytic functions, Oper. Theory Adv. Appl. 43 (1989), 209-216.

8. H. Helson, Large analytic functions 2, in Analysis and partial differential equations (C. Sadosky, ed., Marcel Dekker, Basel, 1990).

9. J. Inoue, An example of a non-exposed extreme function on the unit ball of $H^{1}$, Proc. Edinburgh Math. Soc. 37 (1993), 47-51.

10. M. Lee and D. SARason, The spectra of some Toeplitz operators, J. Math. Anal. Appl. 33 (1971), 529-543.

11. T. Nakazi, Exposed points and extremal problems in $H^{1}$, J. Funct. Anal. 53 (1983), 224-230.

12. T. Nakazi, Sum of two inner functions and exposed points in $H^{1}$, Proc. Edinburgh Math. Soc. 35 (1992), 349-357.

13. T. NAKazi, Extremal problems in $H^{p}$, J. Austral. Math. Soc. Ser. A 52 (1992), 103-110.

14. T. Nakazi and K. Takahashi, Hyponormal Toeplitz operators and extremal problems of Hardy spaces, Trans. Amer. Math. Soc. 338 (1993), 753-767.

15. D. Sarason, Making an outer function from two inner functions, preprint.

Department of Mathematics

FACULTY OF SCIENCE

HOKKAIDO UNIVERSITY

SAPPORO 060

JAPAN 